\title{
Nonlinearity enhances operation of fiber ring laser
}

Ping Shum, Ming Tang, Yandong Gong, Xinyong Dong, Songnian Fu, Hui dong, and Xiufeng Yang

Parametric four-wave mixing in nonlinear fiber allows continuous wave or mode-locked fiber ring lasers to achieve ultrastable, superior performance for high-power multiwavelength output.

Fiber lasers, especially multiwavelength erbium-doped fiber lasers (MW-EDFLs), have attracted considerable interest for potential applications in optical test and measurement, and optical wavelength-division-multiplexing communication and sensing systems. ${ }^{1}$ Compared with compact semiconductor-based lasers, EDFLs are competitive because of their all-fiber structure as well as their capacity to provide high power and ultrashort pulse width. ${ }^{2}$ They can be used in applications that require multiple wavelength sources, with small, equal wavelength spacing, a large number of peaks within a broad band, and high output uniformity across the channels. These requirements pose a very challenging task for building a cost-effective multiwavelength EDFL for continuous wave (CW) or pulsed operation.

Previously, due to the homogeneously broadened gain property, many MW-EDFLs were developed with wavelength spacing larger than homogeneous linewidth $(\sim 3.5 \mathrm{~nm})$ to overcome gain competition. Although a MW-EDFL with 16 wavelengths has been accomplished by cooling an erbium-doped fiber (EDF) to 77K using liquid nitrogen, the increased complexity and cost make it impractical for real applications. Many different approaches have also been explored for developing MW-EDFLs, including using polarization or spatial hole burning, using independent gain media, frequency shifting, and phase modulation. . $^{3-7}$

Here, we demonstrate a novel room-temperature-operated MW-EDFL with wavelength spacing less than homogeneous broadening linewidth, based on interchannel four-wave mixing (FWM). ${ }^{8}$ The gain-clamping effect in EDF is compensated by the parametric FWM between multiwavelength channels in a highly nonlinear fiber section that is inserted into the fiber ring cavity.

First, we implemented a stable CW MW-EDFL based on highly nonlinear photonic crystal fiber (HNL-PCF) and sampled-fiber Bragg grating (SFBG), as described in Figure 1. We

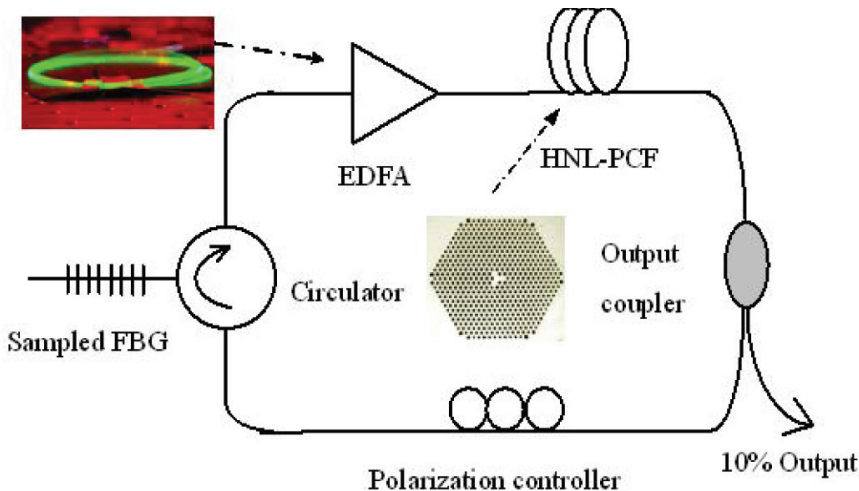

Figure 1. Schematic diagram of the multiwavelength erbium-doped fiber laser. PC: Polarization controller. FBG: Fiber Bragg grating. HNL-PCF: Highly nonlinear photonic crystal fiber. EDFA: Erbiumdoped fiber amplifier.

initiated multiple wavelength operation, as shown in Figure 2, by adjusting the polarization controller and a specific SFBG with $0.8 \mathrm{~nm}$ wavelength spacing. We then used an FWM-induced dynamic gain-flattening mechanism to stabilize the output. The FWM processes suppress the homogeneous line broadening of the EDFL and stabilize the multiple wavelength oscillation. By tuning the intracavity polarization controller and then the FWM efficiency, the number of concurrent lasing wavelengths can be changed from two to five, and the peak power differences for the main oscillation wavelengths are less than $2.0 \mathrm{~dB}$.

We further extend the idea of FWM-induced self-stable operation of MW-EDFL to the multiwavelength mode-locking fiber ring laser to generate ultrashort, ultrafast, multiwavelength and synchronized pulse sources, as shown in Figure 3. By using $1 \mathrm{~km}$ conventional highly nonlinear fiber (HNLF), two and four wavelengths anchored on the International Telecommunications Union standards (ITU-T) with $100 \mathrm{GHz}$ channel spacing are successfully mode-locked at $10 \mathrm{GHz}$ simultaneously, without gain competition.

Continued on next page 


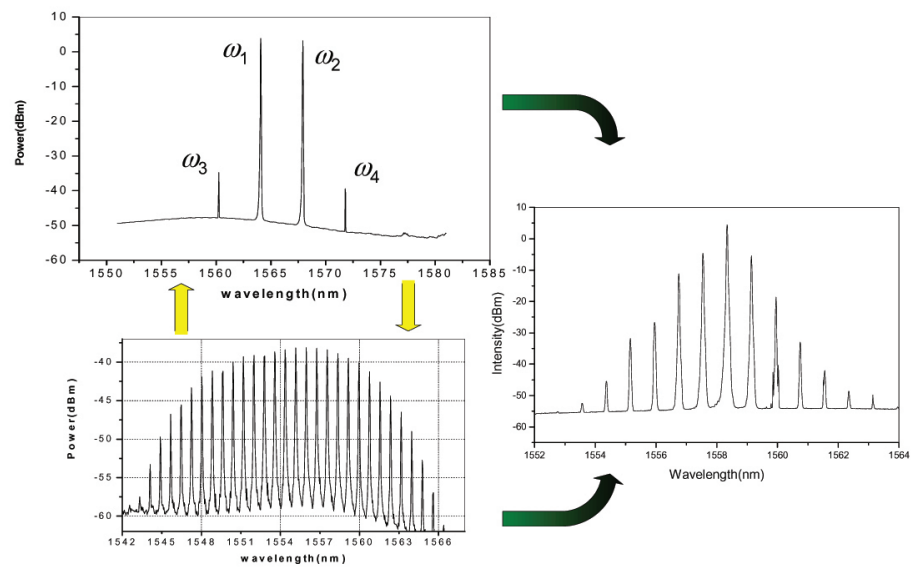

Figure 2. The multipeak transmission spectrum of sampled fiber Bragg grating (SFBG) and the interchannel parametric four-wave mixing (FWM) lead to stable multiwavelength output.

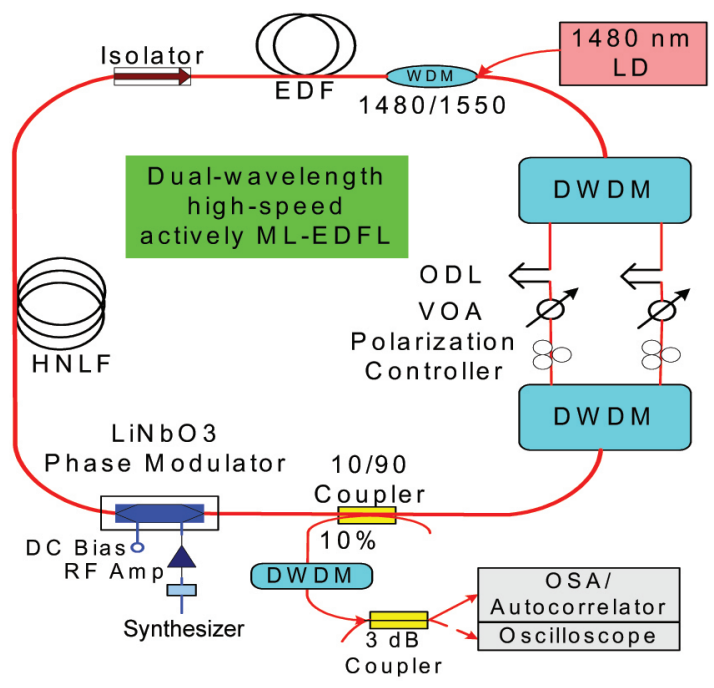

Figure 3. Experimental setup for multiwavelength mode-locked erbium-doped fiber ring laser (ML-EDFL). EDF: Erbium-doped fiber. WDM: Wavelength division multiplexing. LD: Laser diode. HNLF: Highly nonlinear fiber. DWDM: Dense WDM. ODL: Optical delay line. VOA: Variable optical attenuator. RF: Radio frequency. OSA: Optical spectrum analyzer.

Moreover, we implement a much more compact cavity structure with $60 \mathrm{~m}$ HNL-PCF to achieve the extremely small channel spacing of $0.5 \mathrm{~nm}$ operation of $10 \mathrm{GHz}$ dual-wavelength mode locking with the help of fiber birefringence. This is the densest $10 \mathrm{GHz}$ mode-locked fiber laser reported, to the best of our knowledge, and the system can easily be scaled to provide more wavelengths. Figure 4 shows the spectrum and pulse train of two mode-locked wavelengths. The amplitude fluctuation and
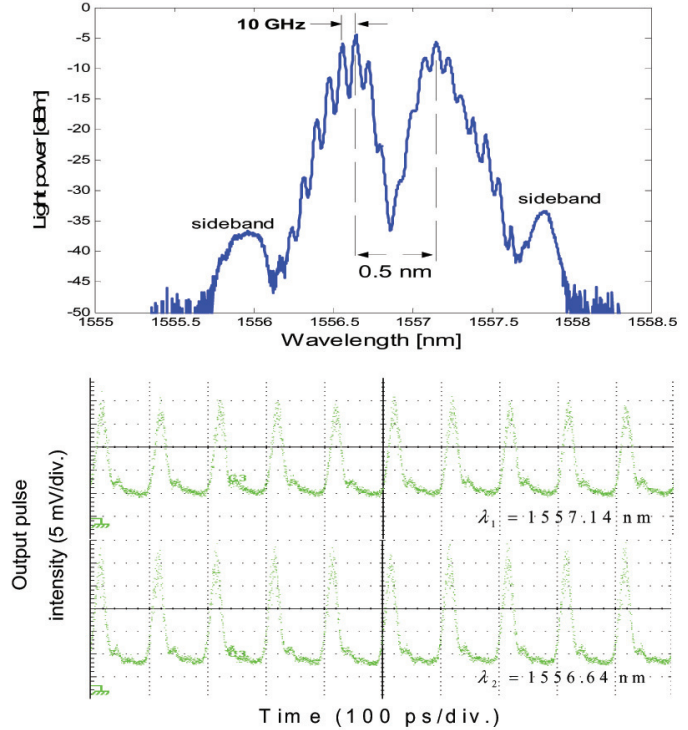

Figure 4. Dual-wavelength mode-locking performance. (a) Optical spectrum. (b) Direct pulse train output.

timing jitter are measured to be less than $1 \%$ and $100 \mathrm{fs}$, respectively. The supermode suppression ratio is higher than $60 \mathrm{~dB}$. The time-bandwidth products vary between $0.39 \sim 0.41$, hence our laser generates nearly transform-limited pulses. Also, the channel spacing can be designed according to the repetition rate and the fiber birefringence evolution.

In conclusion, we proposed and demonstrated a novel roomtemperature MW-EDFL that uses FWM throughout the length of the HNLF to suppress homogeneous gain broadening. The optimal combination of nonlinearity and dispersion of the HNL-PCF enables efficient FWM generation and eliminates the interchannel saturation effect. It is a promising start to building a simple structure incorporating a laser cavity and nonlinear fiber, which can easily generate more wavelengths with dense channel spacing.

\section{Author Information}

Ping Shum, Ming Tang, Songnian Fu, and Hui Dong

Network Technology Research Centre (NTRC)

Nanyang Technological University

Singapore, Singapore

\section{Yandong Gong and Xiufeng Yang}

Institute for Infocomm Research

Singapore, Singapore

Continued on next page 


\section{Xinyong Dong}

Photonics Research Centre

The Hong Kong Polytechnic University

Hong Kong, China

\section{References}

1. D. Wei, T. Li, Y. Zhao, and S. Jian, Multiwavelength erbium-doped fiber ring lasers with overlap-written fiber Bragg gratings, Opt. Lett. (25), pp. 1150-1152, 2000.

2. A. Bellemare, M. Karasek, M. Rochette, S. LaRochelle, and M. Tetu, Room temperature multifrequency erbium-doped fiber lasers anchored on the ITU frequency grid, J. Lightwave Technol. (18), pp. 825-831, 2000.

3. O. Graydon, W. H. Loh, R. I. Laming, and L. Dong, Triple-frequency operation of an Er-doped twin core fiber loop laser, IEEE Photon. Technol. Lett. (8), pp. 63-65, 1996.

4. G. Das and J. W. Y. Lit, L-band multiwavelength fiber laser using an elliptical fiber, IEEE Photon. Technol. Lett. (14), pp. 606-608,2002.

5. C.-L. Zhao, X. Yang, C. Lu, J. H. Ng, X. Guo, P. R. Chaudhuri, and X. Dong, Switchable multi-wavelength erbium-doped fiber lasers by using cascaded fiber Bragg gratings written in high birefringence fiber, Opt. Commun. (230), pp. 313-317, 2004.

6. A. J. Poustie, N. Finlayson, and P. Harper, Multiwavelength fiber laser using a spatial mode beating filter, Opt. Lett. (19), pp. 716-718,1994.

7. Q. Mao and J. W. Y. Lit, Switchable multiwavelength erbium-doped fiber laser with cascaded fiber grating cavities, IEEE Photon. Technol. Lett. (14), pp. 612-614, 2002.

8. G. P. Agrawal, Nonlinear Fiber Optics, 2nd ed., Academic Press, San Diego, CA, 1995. Chapter 10 